\title{
Better and stronger potatoes using hybrid breeding
}

Potato is a hugely important crop worldwide, being eaten across the world and across productivity and crop hed cater for the increasing global population, and also serve to reduce famine in challenging and impoverished environments. The potato breeding company Solynta in The Netherlands has successfully produced a hybrid breeding program for potatoes which will allow the rapid selection and turnover of favourable traits, millions of people worldwide.

otato breeding techniques are relatively few calories, yet potatoes can be lagging far behind those of other and wheat. This is because potato breeding suffers hinderances through its genetic complexity, resulting in slow progress. The cultivated potato Solan tuberosum is tetraploid, meaning it has four sets of chromosomes - in this situation, it is easy for unfavourable alleles to make their way unseen into the next generation, and introducing new favourable traits can be very tricky.

At Solynta, a potato seed breeding company based in Wageningen, Holland, an innovative method for targeted breeding has been developed which will change the production and globa distribution of potatoes. This method allows for the combination of beneficia to make 'elite' progenies which have a higher yield, disease resistance, and of course, better taste. Furthermore, this method allows for vastly improved transpor vastion which will be done at the seed stage, rather than fully grown potato tubers.

Potatoes are also a much more nutritious source other top crops such as rice, soy and wheat. They contain hig
levels of levels of processed and cooked into many diffe forms - potentially providing varieties With the help of the Solynt breeding technology, each of these traits can and reproduced.

\section{A HYBRID BREEDING PROCESS} varieties took a very long time. Firstly, it was simply trial and error to breed a suitably stronger crop, after this the selected plant underwent a lengthy multiplication process. The best potato would be selected and planted in the ground to multiply the number of tubers, this process takes a year. This must be repeated year upon year, until viter around seven years a commercially maters worse, with time, these potatoes can become increasingly susceptible Phytophthora infestans.

This new targeted breeding process relies on a few important principles which counteract these historical problems. The first is that the breeding process is completed using diploid potatoes rather than tetraploid. This is achieved by diploidisation and using S. chacoense diploid potatoes. The resulting organism is a diploid hybrid potato plant, with just two sets of chromosomes. Nornally, diploid potato plants are selfplants cont in some S. chacoense plants contain a gene called
overcomes self-incompatibility. be harnessed genetically selected for, to diseases, such as potato blight

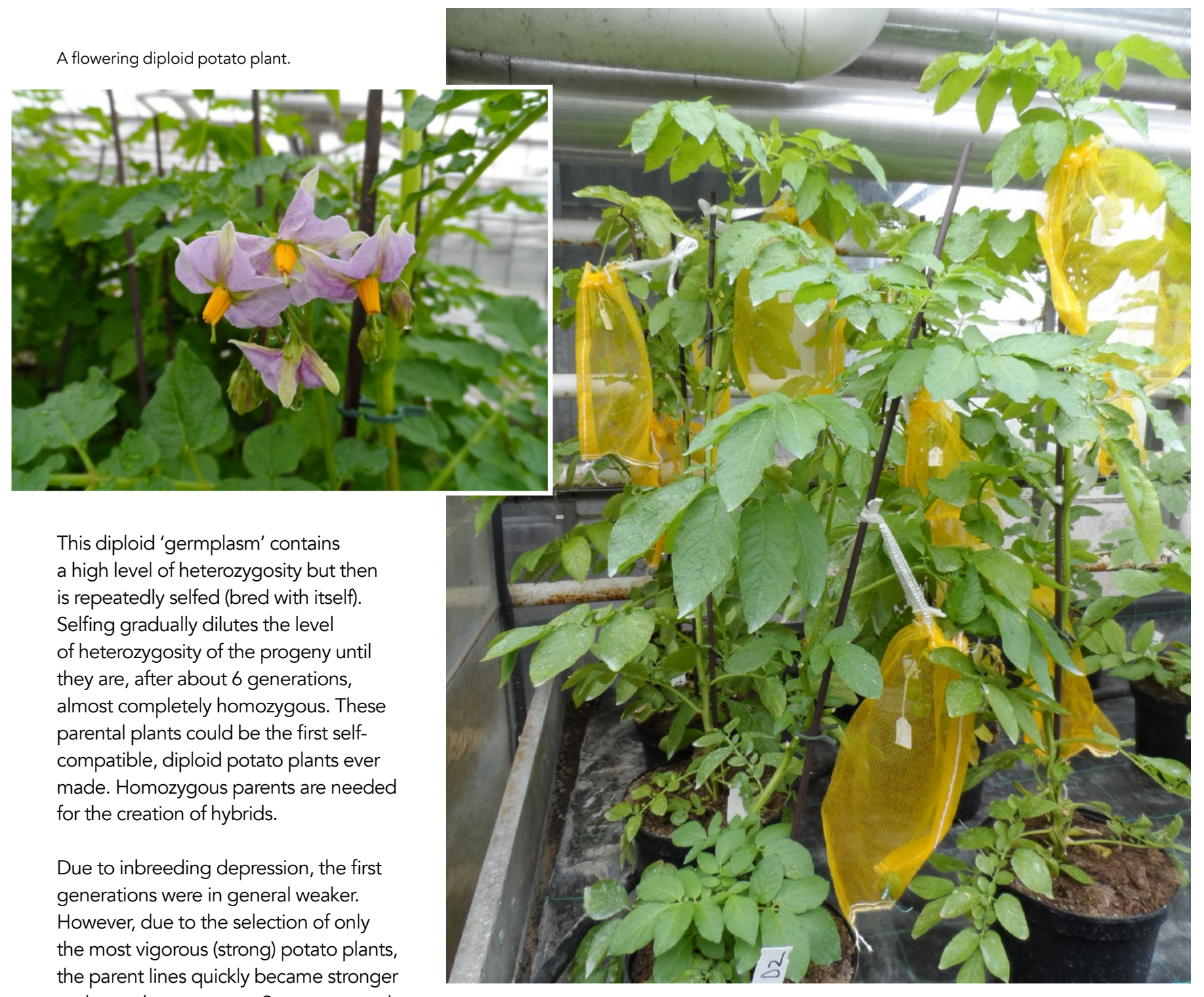

the most vigorous (strong) pot ofly

作 plants can hy hous. Strong parental hybrid seeds. The resulting hybrid potato Berry development after
berries from other crosses plants were generally better and stronger than the inbred parent lines (heterosis).

\section{THE BENEFIT OF SEEDS}

The potato seeds can be harvested when the hybrid plant forms berries. These seeds (true potato seeds) have an incredible benefit over the previous method with tubers (tubers are also named seed potatoes). The weight of 100 potatoes is equal to the weight of 16 million seeds, as it's

drastically reduce transportation cost and difficulty.

The seeds are also pathogen free whereas tubers mostly have a low level of disease-causing pathogens. True potato seeds also withstand changes in temperature and humidity. Ultimately the reduction in transport will also reduce carbon footprint.

DISCOVERING TRAITS,

One huge application of Solynta's potatoes will be to help farmers and feed people, in previously difficult or challenging locations. potato plants is present, Solynta could then focus on isolating and selecting for beneficial potato traits. During this strong pocess, the traits needed for genetically targeted and selected for This was done using a process called OTL (Quantitative Trait Locus) mapping.

Each phenotypic trait (observed in the potato) is described by one or, normally more than one, gene. In order to understand which genes control the outcome of a potato, the team looked at SNPs (Single Nucleotide Polymorphisms). Simply put, this is when one nucleotide differs on a particular individual's genome. By using sequencing and statistics, it's possible particular phenotypic trait. Plants with 


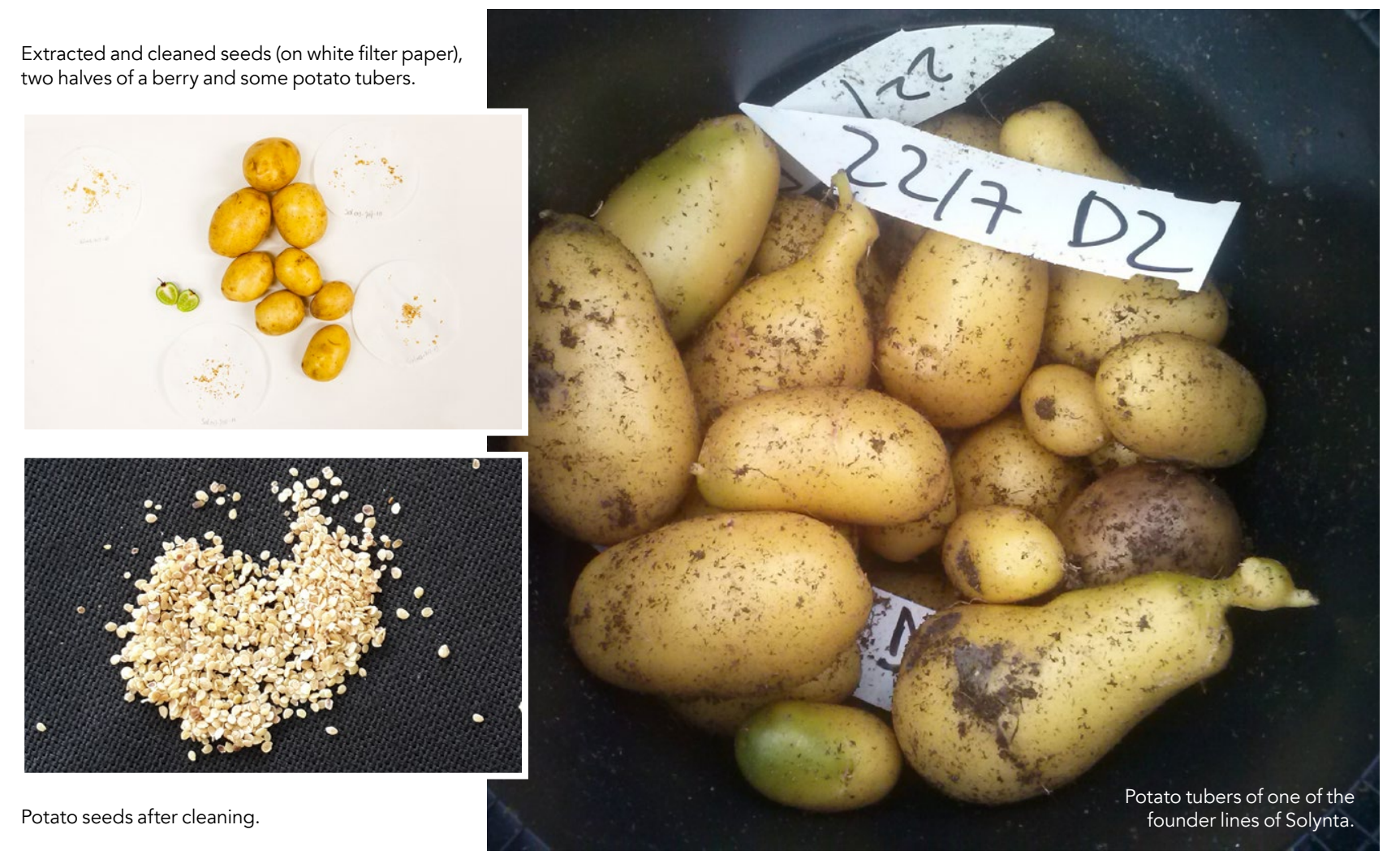

those detected genome alterations are then selected for breeding.

In order to further enhance this process, the parental lines could then be 'backcrossed' to add new 'hybrid traits' to one of the parent lines. This strengthened the parent lines, which were then hybridized to make an even stronge hybrid potato. Each time a stronger, tastier, or larger potato was grown. This
process is so rapid in Solynta because of the speed of growth from seed,

These parental plants could be the first self-compatible, diploid potato plants ever made. Homozygous parents are needed for the creation of hybrids.

\section{FEEDING GLOBAL POPULATIONS but even larger in low to middle-} One huge application of Solynta's income countries. potatoes will be to help farmers and feed people, in previously difficult The Solynta hybrid diploid potato seeds or challenging locations. The benefits of these potatoes are going to be

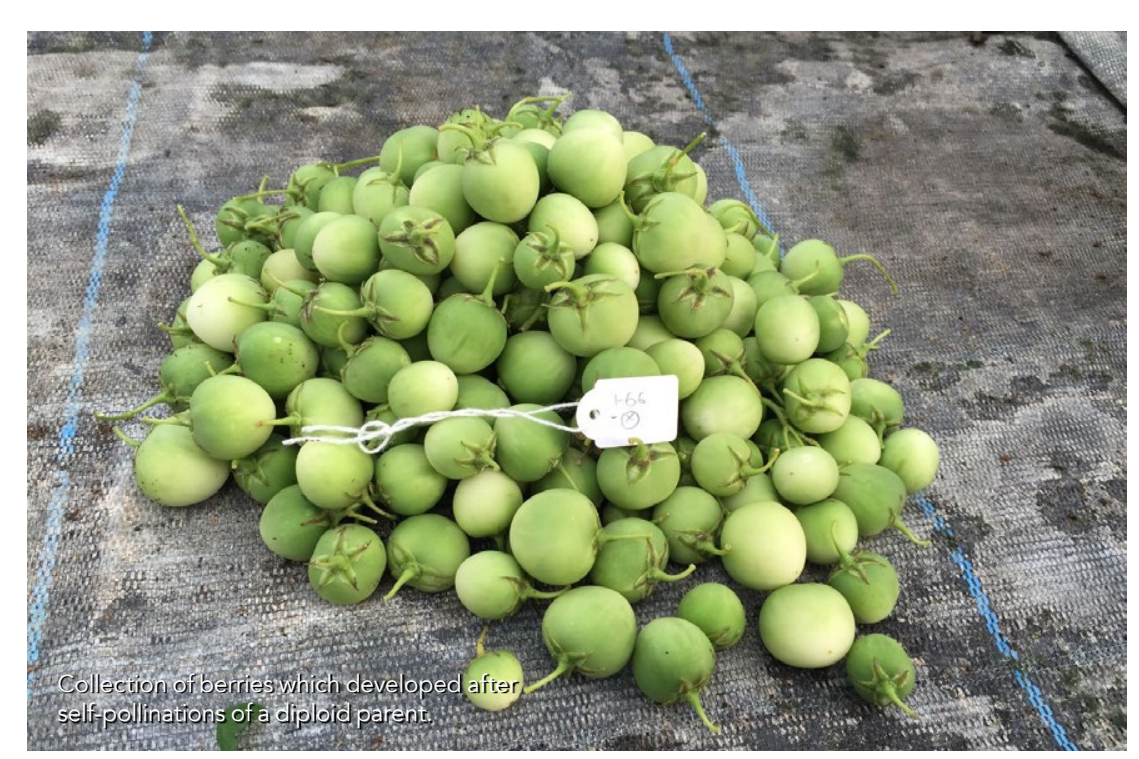
were transported and tested in East DR Congo. The results were extremely optimistic, with a much higher yield of potato than under normal East African conditions.

Furthermore, the seeds were extremely easy to transport and were free of pathogens which are so common in the tropical African climate and can decimate farmers' stocks between seasons. It's also very possible to adapt the diploidisation and genetic hybridization techniques from Solynta's technology to African potato varieties, increasing the breadth of choic in these areas. It seems only a matter of time before these farming methods are being used worldwide in order to catch up with the global demand for food production alongside

\section{Behind the Research} Dr.ir. Sjaak van Heusden

E: sjaak.vanheusden@solynta.com T: +31620843929 W: https://solynta.com/

\section{Research Objectives}

Solynta has developed an innovative method for targeted breeding which will change the production and global distribution of potatoes

\section{Detail}

Dr.ir. Sjaak van Heusden

Solynta

6703HAWAGENINGEN

The Netherlands

Bio

Sjaak van Heusden fields of expertise are tomato and diploid potato pre-breeding with emphasis on marker technology. Besides research, he is involved in international teaching and cooperation. In the last five years, he has been mainly employed as a geneticist at the potato breeding company Solynta.

Funding

Solynta

Collaborators

-Wageningen University and Researc

\section{的olynta...}

\section{References}

De Vries M, ter Maat M, Lindhout P. (2016). 'The potential of hybrid potato for East-Africa'. Open Agriculture, Vol 1: pp. 151-156.

Lindhout $P$, de Vries $M$, ter Maat $M$, Ying $S$, Viquez-Zamora M van Heusden S. (2018). 'Hybrid potato breeding for improved varieties. Ach. pp. 99-122.

Lindhout P, Meijer D, Schotte T, Hutten R, Visser R, van Eck H. (2011). 'Towards F1 Hybrid Seed Potato Breeding'. Potato Research, Vol 54 (4), pp301-312.

D. Meijer; M. Viquez-Zamora; H. J. van Eck; R. C. B. Hutten: Y. Su; R. Rothengatter; R. G. F. Visser; W. H. Lindhout: A. W. van Heusden (2018). 'QTL mapping in diploid potato by using selfed progenies of the cross $S$. tuberosum $\times S$. chacoense'. Euphytica, 214:121

\section{Personal Response}

If already successful in other crops, what has stopped this process being achieved before in potatoes? for decades but was never successful. A better use of make it now possible to make the transition of tetrants, potatoes to diploid potatoes Over time, as we transition to diploid potato breeding, we will be able to undo previous dogmas, such as a high yield is only possible
with tetraploid potatoes.

What are your future plans for research in this area?

III Solynta wants to introduce successful varieties in Africa. To do this large numbers of clean seeds of plants with enhanced levels of resistance are needed. Later, othermark new technologies to overcome self-incompatibility and 